\title{
Removal of Ga Implantation on FIB-prepared Atom Probe Specimens Using Small Beam and Low Energy $\mathrm{Ar}^{+}$Milling
}

\author{
C.S. Bonifacio ${ }^{1}$, K.P. Rice ${ }^{2}$, T.J. Prosa ${ }^{2}$, M. Ray ${ }^{1}$, T.F. Kelly ${ }^{2}$, and P.E. Fischione ${ }^{1}$ \\ 1. E.A. Fischione Instruments Inc., Export, PA, USA \\ 2. CAMECA Instruments Inc., Madison, WI USA.
}

Atom probe tomography (APT) is a powerful characterization technique for obtaining three-dimensional structure and materials composition at the near atomic scale. It is also a complementary with other analysis techniques, such as transmission electron microscopy (TEM). In tandem, the two techniques provide a detailed characterization of structure and chemistry. APT specimens are typically prepared using a dual beam focused ion beam (DB-FIB), which is an efficient tool for removing a substantial amount of material, and in situ electron beam imaging allows more control when shaping the APT specimen tip [1]. However, Ga-induced damage and implantation from FIB milling can result in ambiguous results, especially for $\mathrm{Al} / \mathrm{Al}$ alloys [2] and Ga containing materials [3]. Low energy $(<1$ $\mathrm{keV}) \mathrm{Ar}^{+}$milling has been shown to improve TEM specimen quality by removing Ga damage and implantation from FIB preparation [4-5]. Here, we present the use of small beam $(<1 \mu \mathrm{m})$, low energy $\mathrm{Ar}^{+}$milling for the removal of FIB-induced damage from APT specimens.

Si and Al APT specimens were prepared on a Si half-grid with multiple needle carriers. The needles were prepared in a FIB system [Thermo Fisher] using standard lift-out methods and annular milling at $30 \mathrm{kV}$ [1]. Final cleaning steps were performed using an $\mathrm{Ar}^{+}$milling system [Fischione Instruments] prior to APT acquisition using a LEAP $5000 \mathrm{XR}$ [CAMECA Instruments]. $\mathrm{Ar}^{+}$ions were rastered within a defined area and directed longitudinally at the needle at decreasing milling energies (900 and $500 \mathrm{eV}$ ). The protective Pt cap on the needle was removed by the ion milling system; its back-scattered electron detector was used to monitor the needle shape and size in situ. TEM, energy dispersive X-ray spectroscopy, and APT characterization were performed before and after ion milling to determine the removal of FIB-induced damage.

APT 3D reconstruction of Ga distribution on the Si needle shows Ga mostly concentrated on the tip and sidewalls of the needle (Figure 1) - note that APT only captures an interior sub-volume of the full needle, so the Ga damage on the sides of the needle extend from the reconstruction boundary to the real specimen needle boundary (an additional 20-30 nm). TEM similarly shows that these Ga-containing areas are amorphous and are $50 \mathrm{~nm}$ in thickness at the tip and 20 to $25 \mathrm{~nm}$ in thickness on the needle's sidewall (Figure 2b-c). After ion milling at $900 \mathrm{eV}$, these amorphous layers were significantly reduced to $5 \mathrm{~nm}$ at the tip and less than $1 \mathrm{~nm}$ on the sidewalls (Figure 2b) and the Pt cap completely removed. Furthermore, $\mathrm{Ar}^{+}$milling of the Si needle resulted to a very sharp tip (Figure 2c) which is ideal for APT. APT acquisition and TEM analysis of Ga distribution after argon ion milling are underway. The removal of the FIB-induced Ga damage using argon milling will be applied to the Al APT specimens.

\section{References:}

[1] K Thompson et al., Ultramicroscopy 107 (2007), p. 131.

[2] K Fisher and E Marquis, Microscopy and Microanalysis 22 (2016), p. 692.

[3] F Tang et al., Microscopy and Microanalysis 21 (2015), p. 544.

[4] CS Bonifacio et al., Microscopy and Microanalysis 23 (2017), p. 268. 
[5] P Nowakowski et al., Microscopy and Microanalysis 23 (2017), p. 300.

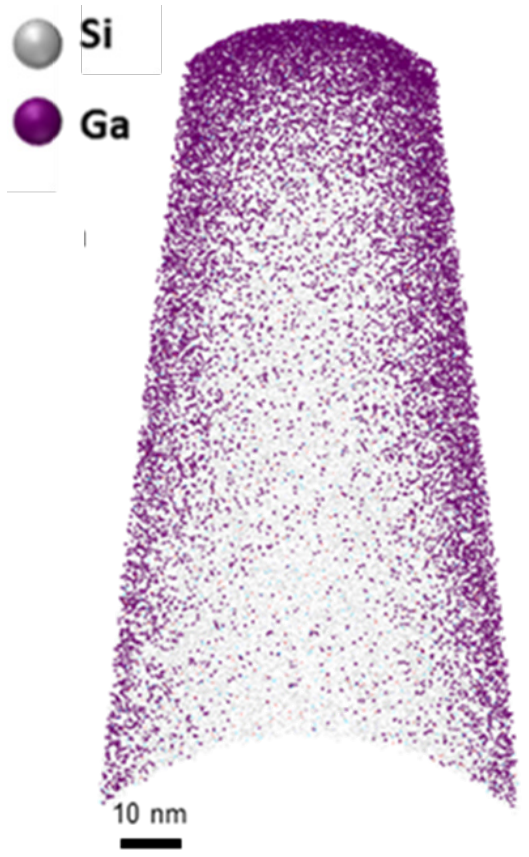

Figure 1. Three-dimensional APT reconstruction of Si APT specimen showing the Ga distribution after $30 \mathrm{kV}$ FIB milling without a cleanup step.
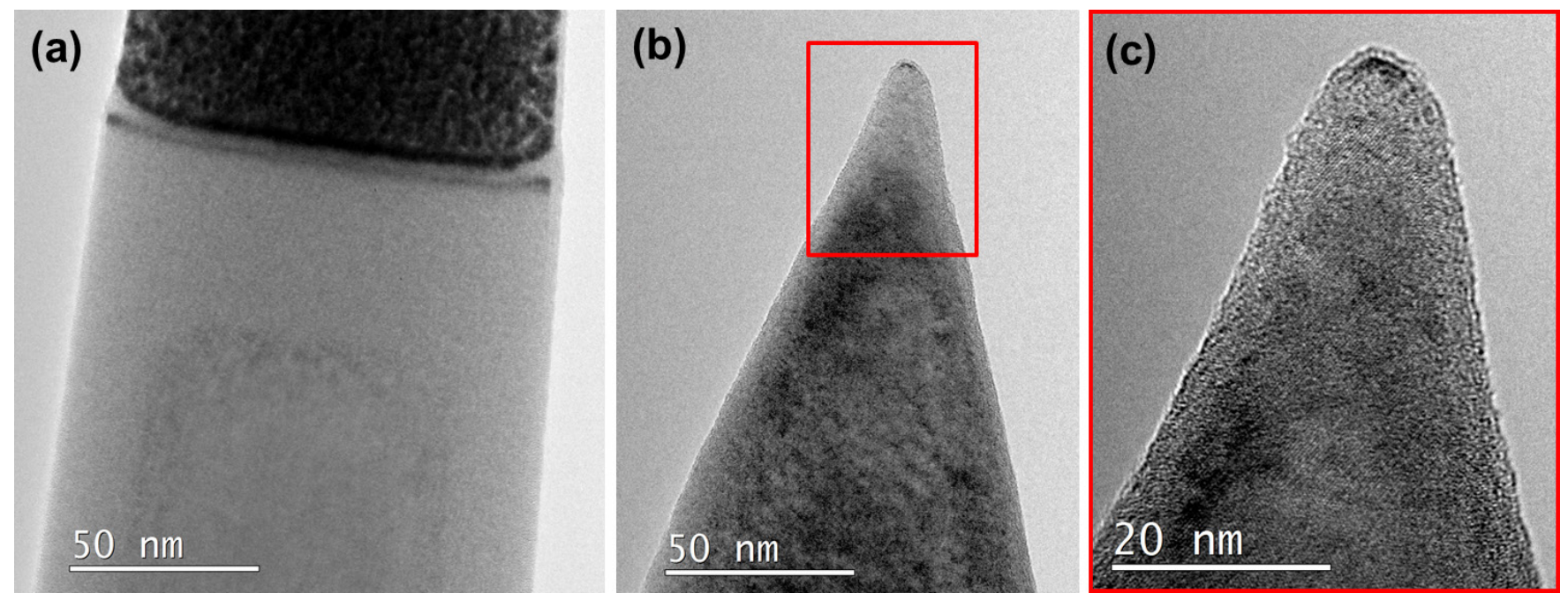

Figure 2. TEM images of the Si APT specimen after $30 \mathrm{kV}$ FIB milling with the Pt protection cap (a) and following $900 \mathrm{eV}$ argon ion milling (b). After ion milling, a significantly reduced amorphous layer and the sharp tip of the needle are seen (c). 